DOI: $10.2478 / \mathrm{v} 10282-012-0010-5$

\title{
Atypical meningioma
}

\author{
G. Iacob, M. Craciun \\ Universitary Hospital Bucharest \\ Motto: "Scientific observation is allways a polemic observation" - Gaston Bachelard
}

\begin{abstract}
Objective: Atypical meningioma are an intermediate group of meningiomas, exhibiting less favourable biological behavior than classic benign tumours, but a relatively more favourable biological behavior than definitive malignant meningiomas. Subject of controversy, atypia in meningioma still generate discordance between accurate criteria defining malignancy and biological behavior, prediction of recurrence.

Methods: This retrospective study intend to evaluate diagnosis on clinical and pathological data, treatment trends and early outcomes for 6 cases with atypical meningiomas occuring in 63 patients operated for benign meningioma in the last 5 years in our clinic between 2006-2011. All patients were explored CT, MRI, preoperative selective angiography and in all cases the WHO 2000 classification criteria were used to define atypical meningioma

Results: Between 2006-2011 we operated 6 atypical meningioma of 63 benign meningiomas $(9,52 \%)$. Tumor sites in the patients were: parasagittal (3 cases), convexity (2 cases), spheno-cavernous (1 case). All patients were operated and dural graft was done to all cases. The extent of surgical resection was Simpson's grade 1 in 2 cases and Simpson's grade 2 in four cases, to which radiation was administered after the first surgery with a dose ranging from
\end{abstract}

52-62 Gy. Regrowth (enlargement of tumour after subtotal resection) was noticed in 2 irradiated cases: one case after 2 years after the first operation, the patient was again operated - pathological diagnosis was malignant meningioma; in another case after 3 years, at operation it was the atypical meningioma. No chemotherapy was used in our cases.

Conclusions: Atypical meningiomas are rare tumors, grow more rapidely, the diagnosis age $\geq 60$ years, several histological criteria can define accurate identification to understand the biology of this group of tumors. Heterogenous contrast enhancement with marked peritumoral edema in neuroimaging are important; cerebral edema has prognostic value and should encourage fundamental and farmacologic research using anti VEGF and somatostatine analogs treatments. Surgery (Simpson grade 1) referring both tumor and dural implant area should be done de novo but also for recurrencies. Radiotherapy still are controversial without proven benefit and chemotherapy without statistic argues to improve quality of life.

Key words: atypical meningioma, malignant meningioma

\section{Introduction}

Despite maningiomas are considered benign tumors, slow growing, very little multiplication of cells, very rarely invade 
the brain tissue, less likely to recur; atypical meningioma remain a controversial topic because of the limited signs of histological anaplasia, lack of universally accepted criteria for grading histological malignancy few large series have been conducted to evaluate the problem of malignancy and because biological behavior, prediction of recurrence is still difficult to predict. Our study present our experience with such tumors between 2006-2011, operated in our clinic.

\section{Materials and methods}

6 cases with atypical meningiomas occuring in 63 patients operated for benign meningioma in the last 5 years in our clinic between 2006-2011 were evaluated pre and postoperatory, also at 1 and 2 years after. All patients were explored CT, MRI, preoperative selective angiography concerning topography, unique or multiple tumors, the presence of irregular margins and fringes, even prominent tumour pannus extending away from the globoid mass, the pattern of enhancement, the degree of peritumoral oedema, calcifications, brain invasion, spontaneous necrosis in the absence of embolization, vascularization. In all cases the WHO 2000 classification criteria were used to define atypical meningioma on pathological data. All patients were operated on once aiming for gross total resection of the tumour as well as its overlying dura. The extent of surgical resection was graded according to Simpson's classification.

\section{Results}

In the last 5 years 63 meningiomas were operated on at Neurosurgery Clinic Universitary Hospital Bucharest; of this 6
(9,52\%) were atypical meningiomas. Patient age were range 53-78, mean age 67; there was four males and two females; no signs of neurofibromatosis type 2 or another associated tumoral pathologies were noticed. At presentation all patients had intracranial hypertension syndrome: headache, papillary oedema, confusion 1 case; also slight hemiparesis or only limb paresis, focal seizures.

Tumor sites in our patients were: parasagittal (3 cases), convexity (2 cases), spheno-cavernous (1 case). Cerebral MRI an CT scans were done in all patients. The tumors were slighty hyperdense with no calcification.

All tumours exhibited homogenous dense contrast enhancement, with marked or moderate peritumoral oedema, irregular tumor margins. Angiography was performed in all cases with homogenous vascular blush, in two convexity meningiomas vascular feeders were seen from external carotid artery suited by embolization. (Figure 1)
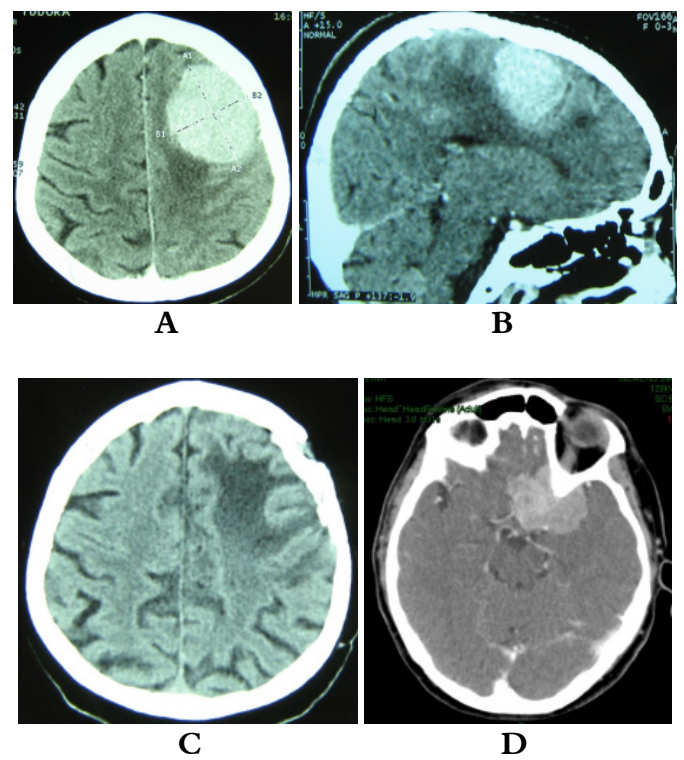


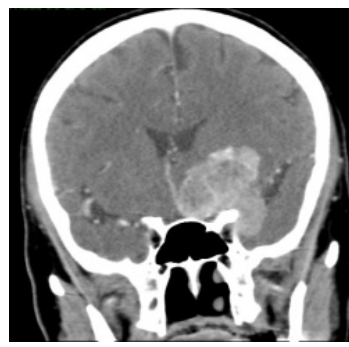

E

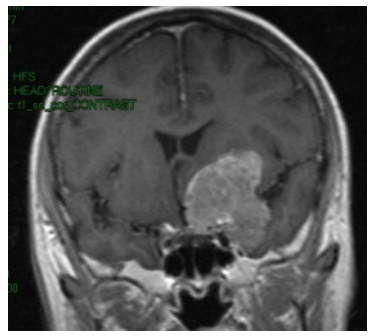

G

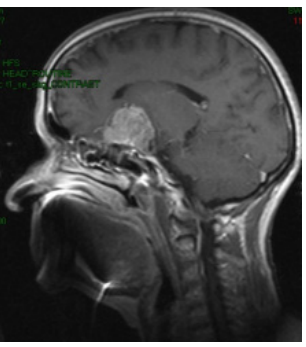

$\mathbf{F}$

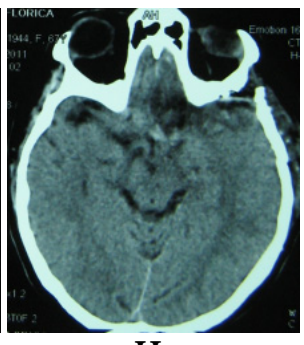

$\mathbf{H}$

Figure 1 A-C pre and postop CT scan in frontal atypical meningioma with important perilesional oedema; D-E pre-op CT scan and F-G MRI of a spenocavernous atypical meningioma; $\mathrm{H}$ post-op CT scan

All patients were operated and dural graft (autologous or synthetic) was done to all cases. The extent of surgical resection was Simpson's grade 1 in 2 cases and Simpson's grade 2 in four cases, to which radiation was administered after the first surgery with a dose ranging from 52-62 Gy.

Pathological characteristics in all cases were based according to the WHO 2000 classification: at least 4 mitoses in $10 \mathrm{hpf}$ or 3 of the following criteria: increased cellularity, high nuclear-to-cytoplasm ratios, prominent nucleoli, uninterrupted patternless or sheet-like growth or necrosis. (Figure 2)

The median follow-up was 26 months (range 12 - 48 months). All patients with atypical meningioma in our series survived. Regrowth (enlargement of tumour after subtotal resection) was noticed in 2 irradiated cases:

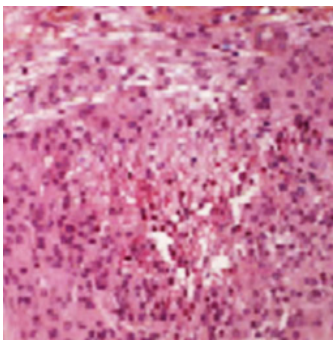

A

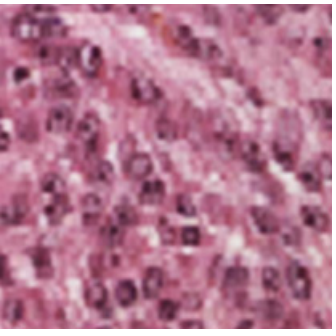

C

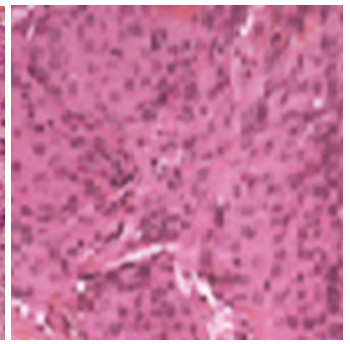

B

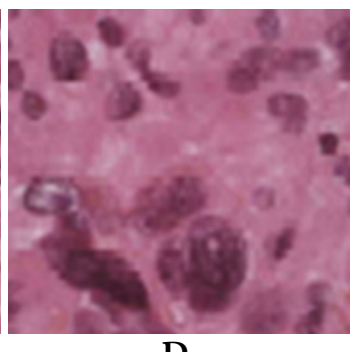

D
Figure 2 Atypical meningioma - pathological characteristics: A. necrosis (HE X 20), B increased cellularity and sheet-like growth (HE X 20), C prominent nucleoli (HE X 40), cellular atypia (HE $\mathrm{X} 40$ )

one case after 2 years after the first operation, the patient was again operated pathological diagnosis was malignant meningioma; in another case after 3 years, at operation it was the atypical meningioma. No chemotherapy was used in our cases.

\section{Discussion}

Usually benign, recognized as a clinical entity for nearly 200 years, arising from arachnoidal cells of neural crest (1)(2)(3), meningiomas are the most common, primary, intracranial extraaxial brain tumours and comprise $13-20 \%$ of all primary intracranial neoplasms; they can invade dura and the skull by osseous hyperplasia (4). Acordind to the WHO

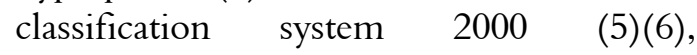
meningiomas are regarded as a heterogeneous group of tumours and are 
histologically categorized into 14 distinct subgroups with three grades of malignancy:

- benign (Grade I - typical meningiomas) - 90\%: meningothelial, fibrous, transitional, psammomatous, microkystic, angioblastic (most aggressive), metaplasic; these tumours exhibit slow growth and very little multiplication of cells and very rarely invade the brain tissue. Overall, benign meningiomas are less likely to recur than the atypical and malignant grades.

- atypical (Grade II- M2, atypical) - 5\%$10 \%$ : atypical variants of their original histologic types: meningothelial, fibroblastic, transitional, including brain invasion, chordoid (foci of chordoid similar to a chordoma), clear cell (poligonal cells with clear cytoplasma by glicogen accumulation). It's to be remarked that dura and adjacent bone invasion are not atypia signs. These tumours exhibit increased cellularity, cell abnormalities: prominent nucleoli, small cells, modified cell architecture, spontaneous necrosis in the absence of embolisation, a faster growth rate than benign meningiomas, some degree of brain invasion and a higher likelihood of recurrence than benign.

- anaplastic (Grade III - M3, malignant) - 3-5\% of all meningiomas: - papillary, rhabdoid, anaplastic. These tumours show frequent mitoses, increased cellular abnormalities, high nuclear to cytoplasmic ratio, foci of necrosis; exibit a faster growth rate compared to benign and atypical meningiomas, recur and are the most likely to invade the brain and spread (metastasize) to other organs in the body.

Accordind to WHO 2000 classification (5) atypical meningiomas have at least: 3 of the 5 following criteria: increased cellularity, high nuclear to cytoplasm ratios, prominent nucleoli, uninterrupted patternless or sheet-like growth or necrosis; also 4 mitoses in $10 \mathrm{hpf}(\mathrm{x} 400)$. The new WHO 2007 classification mentain the ancient classification, for atypical meningioma; brain invasion is not a criteria, but is recognised as an evolutivity criteria (7). Although recognised as as pronostic factors of agresivity: brain invasion, elevated MIB-1 labeling (8)(9) and Ki67 proliferative cells are not criteria for atipic meningioma (10), despite recognized predisposition for tumor recurrence and overal survival (10) when these factors are noted. Nagashima (11)(12) has noted cmyc expression level, the protooncogene and his protein proving positive correlation between c-myc expression level and the proliferative cell index by anti Ki67 immunomarking. Their etiology is uncertain, but typical meningiomas may undergo gene losses and gains, transforming them into atypical ones (13). Radiation-induced meningiomas are also more likely to be atypical (14)

Atypical meningiomas are rare, more aggressive and invasive than typical meningiomas, grow more rapidely, become symptomatic at an earlier age, are predominant in adults (2)(10)(15-21). Interesting especially the 4th and 5th decade, age egal or over 60 years are a negative prognostic facto in multivariants analisis. Considering gender distribution, asa in our cases, the female prodominance of $2 / 1$ seen in benign meningiomas are no more observed, there is a inversion tendency for men for meningioma progressing from atypical to malignant meningioma, even biochemical studies have shown that progesterone activity correlates inversely with atypical in meningiomas.

Clinical features are not specific, but as a 
DOI: $10.2478 / \mathrm{v} 10282-012-0010-5$

reflection of increased peritumoral oedema in atypical meningioma are more likely related with objective neurological deficits (22). More common atypical meningioma occur over cerebral convexities, parasagittal and falx;, few reports identify other locations as: infratentorially, supra and infratentorial extensions, intraventricularly (2).

CT as MRI could not be an indicative to differentiate benign from atypical meningiomas, also to predict aggressive potential. Atypical meningiomas may be plaquelike in configuration and extend through the dura to the skull and scalp or can spread via the Perivascular Spaces (14)

Marked peritumoral oedema, heterogenous contrast enhancement in $5,4 \%$, minimal or calcifications absence, irregular or indistinct margins, musjroomlike projections of the main tumor mass are the most important findings in atypical meningiomas. (2)(15)(20)(22-24). DWI (diffusion-weighted imaging) signal intensity of tumors classified as hypo-, isoor hyper-intense to grey matter and apparent diffusion coefficient (ADC) measurements to differentiate benign from atypical/malignant meningiomas and among different sub-types do not seem reliable in grading meningiomas or identifying histological sub-types. Hence, these parameters should not be recommended for surgical or treatment planning.(6) Concerning cerebral oedema in atypical meningiomas several considerations could be stated: there is no correlation between peritumoral oedema and tumor grade in meningioma (25), a significant relation was statued between microvascular density, the value of the vascular endothelial growth factor expression (VEGF) known also as a vascular permeability factor and some somatostatine receptors (sst2) (26). Somatostatine by his receptors has an antiproliferative activity on several cells (27) for this reason these receptors offers new tendency for research in tumor recurrency treatment.

In the absence of a prospective study concerning the surgery effect on meningioma (28) The best attitude still is the surgical approach with large dural implant base resection, to reduce recurrency and mortality.(2)(19)(29) Using a univariate analysis Palma (16) has proved that Simpson 1 rezection versius 2-3 can improve global survival for atypical meningiomas $(\mathrm{P}<0,0071)$, especially when tumor is on convexity. To improve tumor resection several methods were used: neuronavigation, somatostatine analogs used intraoperatory for a better identification (30), specific imaging octreoscan, microsurgery (6).

Radiation therapy in the treatment of atypical meningioma still are controversial (2)(31). Despite classical conception of meningioma radioresistance, radiotherapy was used indifferent of the extent of surgical resection, the mean dosis 53,57 gray +/_6,06 grays. On retrospective studies radiotherapy has doubtful results without consensus: some authors recommend radiotherapy after the first recurrency $60,5 \%$, another authors proved that radiotherapy has no impact on general survival and even can deteriorate prognosis. Radiotherapy should be proposed especially when tumor is passing from grade 2 to 3 . Radiotherapy did no prevent or retard the recurrence of tumours regardless of the extent of resection (31). No chemotherapy is usefull (6). Despite gross total resection, approximately $29-40 \%$ of atypical meningiomas recur and survival of higher 
grade tumours correlated with specific histological features have produce conflicting data (21).

\section{Conclusions}

Atypical meningiomas are rare tumors, with uncertain etiology, grow more rapidely, the diagnosis age $\geq 60$ years, several histological criteria can define accurate identification to understand the biology of this group of tumors. Heterogenous contrast enhancement with marked peritumoral edema in neuroimaging are important; cerebral edema has prognostic value and should encourage fundamental and farmacologic research using anti VEGF and somatostatine analogs treatments. Surgery (Simpson grade 1) referring both tumor and dural implant area should be done de novo but also for recurrencies. Radiotherapy still are controversial without proven benefit and chemotherapy without statistic argues to improve quality of life.

\section{References}

1. Claus E.B. et al. - Epidemiology of intracranial meningioma, Neurosurgery 2005, 57 (6), 1088-95;

2. Sakr S.A., Salem M. - Atypical meningioma: Clinicopathological analysis of a new classification, Pan Arab Journal of Neurosurgery 2011, 15, $136-41$;

3. Cushing H.W., Eisenhardt L. - Meningiomas: Their Classification, Regional Behavior, Life History and Surgical End Results, Springfield, Ill: Charles C. Thomas, 1938, 47-50;

4. Maier H., Öfner D., Hittmair A., et al. - Classic, atypical, and anaplastic meningioma: three histopathological subtypes of clinical relevance, J Neurosurg. 1992, 77:616-623;

5. Louis D.N., et al. - Meningiomas. World Health Organization Classification of Tumours, Pathology and genetics. Tumour of the nervous system 2000, 175-184; 6. Durand Anne - Meningiomes de grade II et de grade III: Etude des facteurs pronostiques, These 2007, Universite de Limoges;

7. Louis D.N. et al. - WHO Classification of Tumours of the Central Nervous System, Fourth Edition, IARC
WHO Classification of Tumours 2007, Acta Neuropathol 2007, 114: 97-109;

8. Modha A., Gutin P.H. - Diagnosis and treatment of arypical and anaplastic meningiomas: a review, Neurosurgery, 2005, 57(3), 538-550; discussion 538550 ;

9. Schiffer D., Ghimenti C., Fiano V. - Absence of histological signs of tumor progression in recurrences of completely resected meningiomas, J. Neurooncol. 2005, 73(2), 125-130;

10. Bruna J. et al. - Ki-67 proliferative index predicts clinical outcome in patients with atypical or anaplastic meningioma, Neuropathology 2007, 27(2), 114-120;

11. Nagashima G., et al. - Different distribution of cmyc and MIB-1 positive cells in malignant meningiomas with reference to TGFs, PDGF and PgR expression, Brain Tumor Pathol, 2001, 18(1), 1-5;

12. Nagashima G., et al. - Involvement of disregulated c-myc but not c-sis/PDGF in atypical and anaplastic meningiomas, Clin Neurol Neurosurg. 2001, 103 (1),13-18;

13. Wrobel G, et al. - Microarray-based gene expression profiling of benign, atypical and anaplastic meningiomas identifies novel genes associated with meningioma progression". Int. J. Cancer 2005, 114 (2): 249-256;

14. Russ E., Castello M. - Atypical Meningioma Spreading via the Perivascular Spaces American Journal of Roentgenology, 2002, 178, 3, 768-769;

15. Alvarez F., Roda J.M., Romero M.P et al. Malignant and atypical meningiomas: A reappraisal of clinical, histological and computed tomographic features, Neurosurg. 1987, 20(5), 688-694;

16. Palma L.et al. - Long-term prognosis for atypical and malignant meningiomas: a study of 71 surgical cases, J.Neurosurg. 1997,86(5), 793-800;

17. Ko K.W. et al. - Relationship between malignant subtypes of meningioma and clinical outcome, J.Clin Neurosci 2007, 14(8), 747-753;

18. Joseph E., Sandhyamani S. et al. - Atypical meningioma: a clinicopathological analysis, Neurol India 2000; 48:338;

19. Kepes JJ: Comment on Mahmood A, Caccamo DV, Tomecek FJ, et al. - Atypical and malignant meningiomas: a clinicopathological review, Neurosurgery 1993, 33:963;

20. Mahmood A., Caccamo D.V., Tomecek F.J., et al. Atypical and malignant meningiomas: a clinicopathological review, Neurosurgery 1993, 33:955963;

21. Yang S.Y. et al. - Atypical and anaplastic meningiomas: prognostic implications of clinicopathological features, J. Neurol Neurosurg Psychiatry. 2008, 79(5): 574-80;

22. Joseph E, et al. - Atypical meningioma: a clinicopathological analysis, Neurol India 2000;48:338 
DOI: $10.2478 / \mathrm{v} 10282-012-0010-5$

23. Buetow M.P. et al. - Typical, atypical, and misleading features in meningioma, Radiographics 1991, 11 (6): 1087-106;

24. Jääskeläinen J, Haltia M, Servo A. - Atypical and anaplastic meningiomas: radiology, surgery, radiotherapy and outcome, Surg Neurol 1986, 25:233242;

25. Lamszus K. - Meningioma pathology, genetics and biology, J. Neuropathol Exp Neurol 2004, 64 (4), 275286

26. Pistolesi S. et al. - The role of somatostatin in vasogenic meningioma associated brain edema, Tumori 2003, 89(2), 136-140;

27. Dutour A. et al. - Expression of somatostatin receptor subtypes in human brain tumors, Int.J.Cancer 1998, 76(5), 620-627;
28. Weber D.C. - Adjuvant postoperative high-dose radiotherapy for atypical and malignant meningioma: a Phase II and observation study 2006;

29. DelaMonte S.M., Flickinger J., Linggood R.M. Histopathological features predicting recurrence of meningiomas following subtotal resection, Am J Surg Pathol 1986, 10:836-843;

30. Gay E. et al. - Intraoperative and postoperative gamma detection of somatostatin receptors in bone invasive en plaque meningiomas, Neurosurgery 2005, 57 (1Suppl), 107-113; discussion 107-113;

31. Combs S.E. et al. - Treatment of patients with atypical meningiomas Simpson grade 4 and 5 with a carbon ion boost in combination with postoperative photon radiotherapy: The MARCIE Trial, BMC Cancer 2010, 10:615 doi:10.1186/1471-2407-10-615. 